\title{
Usefulness of confocal laser endomicroscopy for the diagnosis of ileocecal lymphoma
}

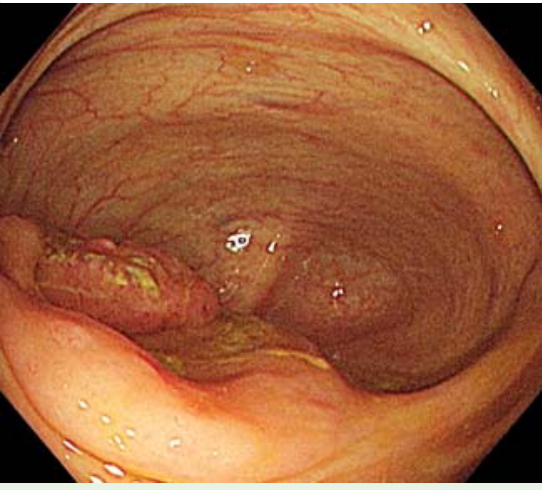

Fig. 1 Conventional endoscopy image. A tumor was identified on the ileocecal valve.

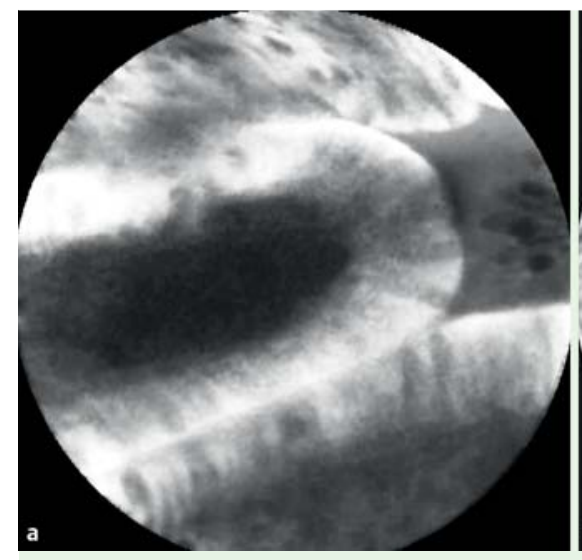

Fig. 2 Probe-based confocal laser endomicroscopy ( $p C L E)$ images. a pCLE showed uniformly villiform architecture on normal intestinal mucosa. $\mathbf{b}$ pCLE showed numerous small cells with destruction of normal mucosal structure on the tumor. Kea Technologies, Paris, France).

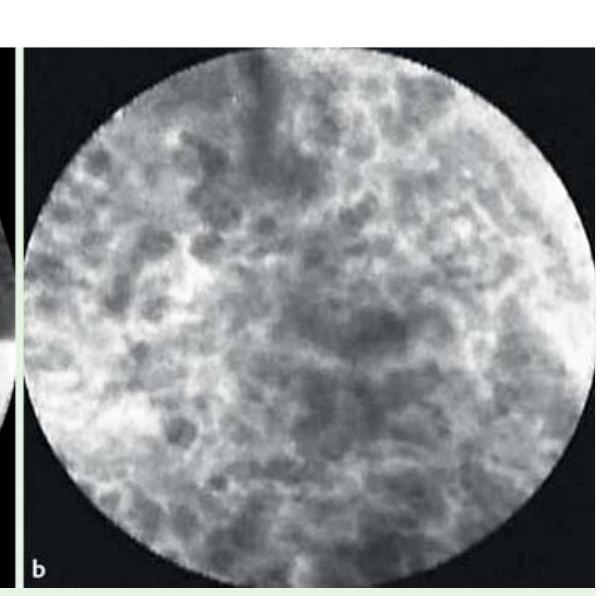

It is often difficult to diagnose malignant lymphoma in the gastrointestinal tract using endoscopy. We report the case of ileocecal lymphoma diagnosed by probebased confocal laser endomicroscopy (pCLE) (ColoFlex UHD, Cellvizio; Mauna

The patient was a 52-year-old man who had a positive fecal occult blood test. He underwent colonoscopy and a tumor was found on the ileocecal valve ( $\bullet$ Fig. 1). A malignant tumor was suspected and a biopsy was taken. The lesion revealed high lymphocyte infiltration, but malignant lymphoma could not be diagnosed.

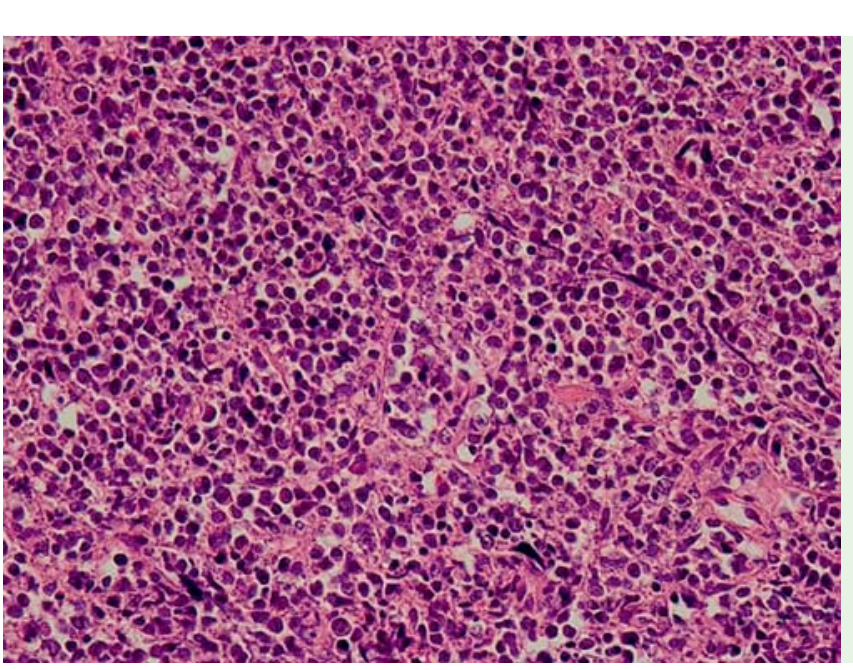

Further multiple biopsies were taken, but a diagnosis could still not be reached. Subsequently, fluorescein-dripping pCLE [1] was performed.

Within the lesion, pCLE identified numerous small cells with destruction of normal mucosal structure, which was similar to the image of malignant lymphoma that we have observed previously ( Fig. 2 , - Video 1) [2]. Polypectomy was performed, and histopathological assessment showed hyperplasia of moderate-to-large sized atypical lymphocytes ( $\bullet$ Fig. 3 ). The cells were immunohistochemically positive for CD20, CD79a, bcl-2, and bcl-6, and negative for CD3, CD5, CD10, and cyclin D1, which resulted in the pathological diagnosis of diffuse large cell lymphoma. The patient was diagnosed as stage I diffuse large cell lymphoma. The patient subsequently received chemotherapy.

As the tumor cell size in the histopathological assessment was close to that of the small cells in the CLE image, we believe that what we observed using pCLE were the lymphoma cells.

\section{Endoscopy_UCTN_Code_CCL_1AC_2AH}

\section{Competing interests: None}

\section{Maiko Takita', Ken Ohata', Kouichi Nonaka', Shinichi Ban², Nobuyuki Matsuhashi ${ }^{1}$}

${ }^{1}$ Department of Gastroenterology, NTT Medical Center Tokyo, Tokyo, Japan 2 Department of Pathology, Dokkyo Medical University Koshigaya Hospital, Koshigaya, Saitama, Japan

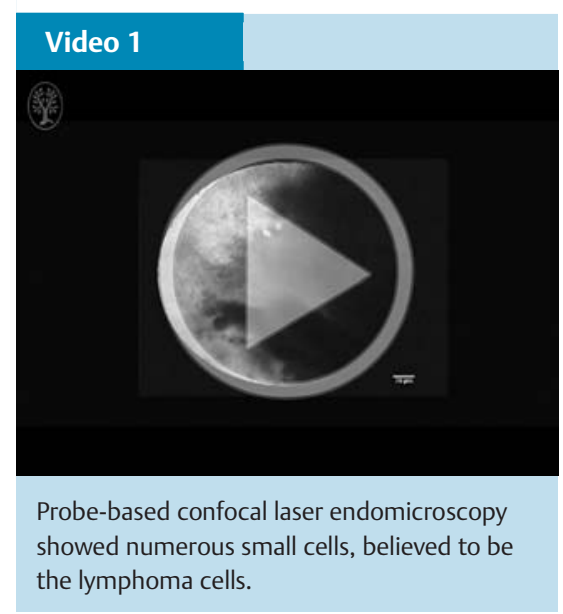




\section{References}

1 Nonaka $K$, Ohata $K$, Nakai Y. Probe-based confocal laser endomicroscopy of the duodenal mucosa with fluorescein dispersion. Dig Endosc 2014; 26: 604

2 Nonaka K, Ohata $K$, Ban S. In vivo imaging of duodenal follicular lymphoma with confocal laser endomicroscopy. Endoscopy 2015; 47 (Suppl. 01): E16-17
Bibliography

DOI http://dx.doi.org/

10.1055/s-0042-105647

Endoscopy 2016; 48: E164-E165

(c) Georg Thieme Verlag KG

Stuttgart · New York

ISSN 0013-726X
Corresponding author

Kouichi Nonaka, MD

Department of Gastroenterology

NTT Medical Center Tokyo

5-9-22 Higashi-gotanda Shinagawa-ku

Tokyo 141-8625

Japan

Fax: +81-3-34486541

nonaka513@gmail.com 